\title{
HOMOTOPY-COMMUTATIVE $H$-SPACES
}

\author{
JAMES P. LIN AND FRANK WILLIAMS \\ (Communicated by Frederick R. Cohen)
}

\begin{abstract}
Let $X$ be an $H$-space with $H^{*}\left(X ; Z_{2}\right) \simeq Z_{2}\left[x_{1}, \ldots, x_{d}\right] \otimes$ $\Lambda\left(y_{1}, \ldots, y_{d}\right)$, where $\operatorname{deg} x_{i}=4$ and $y_{i}=\operatorname{Sq}^{1} x_{i}$. In this article we prove that $X$ cannot be homotopy-commutative. Combining this result with a theorem of Michael Slack results in the following theorem: Let $X$ be a homotopycommutative $H$-space with mod 2 cohomology finitely generated as an algebra. Then $H^{*}\left(X ; Z_{2}\right)$ is isomorphic as an algebra over $A(2)$ to the $\bmod 2$ cohomology of a torus producted with a finite number of $C P(\infty)$ s and $K\left(Z_{2^{r}}, 1\right)$ s.
\end{abstract}

\section{INTRODUCTION}

In this article we prove the following theorem:

Theorem A. Let $X$ be an $H$-space with

$$
H^{*}\left(X ; Z_{2}\right)=Z_{2}\left[x_{1}, \ldots, x_{d}\right] \otimes \Lambda\left(y_{1}, \ldots, y_{d}\right),
$$

where $\operatorname{deg} x_{i}=4$ and $y_{i}=\operatorname{Sq}^{1} x_{i}$. Then $X$ cannot be homotopy-commutative.

The significance of Theorem A lies in its relationship to the following theorem, due to Michael Slack:

Theorem (Slack). Let $X$ be a homotopy-commutative $H$-space with $\bmod 2$ cohomology finitely generated as an algebra. Then

(1) All even-degree generators have infinite height and are in degrees two and four.

(2) All odd-degree generators lie in degrees one and five. The one-dimensional generators have infinite height and the five-dimensional generators are exterior.

(3) $\mathrm{Sq}^{1}: Q H^{4}\left(X ; Z_{2}\right) \rightarrow Q H^{5}\left(X ; Z_{2}\right)$ is an isomorphism.

Received by the editors February 27, 1990.

1980 Mathematics Subject Classification (1985 Revision). Primary 55P45, 55S40.

Key words and phrases. Homotopy-commutative $H$-space, cohomology operation, Steenrod algebra.

The first author's work was supported by a grant from the National Science Foundation. 
Combining Theorem A with Slack's theorem results in the following:

Theorem B. Let $X$ be a homotopy-commutative $H$-space with $\bmod 2$ cohomology finitely generated as an algebra. Then $H^{*}\left(X ; Z_{2}\right)$ is isomorphic as an algebra over $A(2)$ to the mod 2 cohomology of a torus producted with a finite number of $C P(\infty)$ s and $K\left(Z_{2^{r}}, 1\right)$ s.

This theorem extends work of Hubbuck and Lin [3, 4] which proves any finite homotopy-commutative $H$-space has the homotopy type of a torus. Here we treat the larger class of $H$-spaces whose cohomology is finitely generated as an algebra.

Theorem A also has the following immediate corollary:

Corollary C. The 3-connective cover of $S^{3}, \widehat{S}^{3}$, is not $\bmod 2$ homotopy-commutative, hence neither is any product of $\widehat{S}^{3} \mathrm{~s}$ homotopy-commutative.

Remarks. (1) Corollary $\mathrm{C}$ can also be proved by using results in [8] and verifying that the Samelson product $\langle\alpha, \alpha\rangle \neq 0$, where $\alpha$ is a generator of $\pi_{4}\left(\widehat{S}^{3}\right)$. This approach was suggested to the authors by Chuck McGibbon. (The referee has observed that Corollary $\mathrm{C}$ can also be obtained quickly from results of Zabrodsky.)

(2) Our original proof of Theorem A rested upon an analysis of a partial Postnikov tower for $X$. The proof we give here is shorter and is based on a factorization of $\mathrm{Sq}^{8} \mathrm{Sq}^{4}$ (Theorem 1.1 below) that may well be of independent interest.

(3) In the rest of this paper, cohomology will be taken to have coefficients in $Z_{2}$ unless otherwise specified.

\section{Factorization of $\mathrm{Sq}^{8} \mathrm{Sq}^{4}$}

Theorem 1.1. Let $x \in H^{8}(X ; Z)$ be such that $\rho(x)$ lies in the kernel of $\mathrm{Sq}^{2}$, where $\rho: H^{*}(X ; Z) \rightarrow H^{*}\left(X ; Z_{2}\right)$ is reduction $\bmod 2$. Then there is a formula

$$
\mathrm{Sq}^{8} \mathrm{Sq}^{4} x=\mathrm{Sq}^{4} \tilde{\varphi}_{0,3}(x)+\left(\mathrm{Sq}^{9}+\mathrm{Sq}^{7,2}\right) \varphi_{1,1}(x)+x\left(\mathrm{Sq}^{4} x\right)
$$

where the secondary operations $\tilde{\varphi}_{0,3}$ and $\varphi_{1,1}$ are defined by the following relations:

$$
\begin{aligned}
& \tilde{\varphi}_{0,3}: \mathrm{Sq}^{9}=\left(\mathrm{Sq}^{7}+\mathrm{Sq}^{4,2,1}\right) \mathrm{Sq}^{2} \text { on an integral class } \\
& \varphi_{1,1}: \mathrm{Sq}^{2} \mathrm{Sq}^{2}=0 \text { on an integral class. }
\end{aligned}
$$


Proof. Consider the following two-stage Postnikov system.

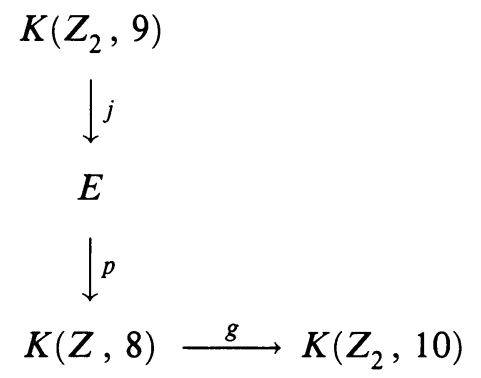

in which $g^{*}\left(l_{10}\right)=\mathrm{Sq}^{2} l_{8}$. Then there are elements $\tilde{v}_{0,3} \in H^{16}(E)$ and $v_{1,1} \in$ $H^{11}(E)$ such that

$$
j^{*}\left(\tilde{v}_{0,3}\right)=\left(\mathrm{Sq}^{7}+\mathrm{Sq}^{4,2,1}\right) l_{9}
$$

and

$$
j^{*}\left(v_{1,1}\right)=\operatorname{Sq}^{2} l_{9} .
$$

Further, $\bar{\Delta}\left(\tilde{v}_{0,3}\right)=p^{*}\left(l_{8}\right) \otimes p^{*}\left(l_{8}\right)$, where $\bar{\Delta}$ denotes the reduced diagonal. We compute

$$
j^{*}\left(\left(\mathrm{Sq}^{9}+\mathrm{Sq}^{7,2}\right) v_{1,1}+\mathrm{Sq}^{4} \tilde{v}_{0,3}\right)=0
$$

and

$$
\bar{\Delta}\left(\left(\mathrm{Sq}^{9}+\mathrm{Sq}^{7,2}\right) v_{1,1}+\mathrm{Sq}^{4} \tilde{v}_{0,3}\right)=\bar{\Delta}\left(p^{*}\left(l_{8}\right) \mathrm{Sq}^{4} p^{*}\left(l_{8}\right)\right)
$$

Hence

$$
z=\left(\mathrm{Sq}^{9}+\mathrm{Sq}^{7,2}\right) v_{1,1}+\mathrm{Sq}^{4} \tilde{v}_{0,3}-p^{*}\left(l_{8}\right) \mathrm{Sq}^{4} p^{*}\left(l_{8}\right)
$$

is an element of $P H^{20}(E) \cap \operatorname{ker}\left(j^{*}\right)$. So $z \in p^{*} P H^{20}(K(Z, 8))$, and thus by [2] $z=c \mathrm{Sq}^{8,4} p^{*}\left(l_{8}\right)$, for some $c \in Z_{2}$.

To prove the theorem, we must show that $c=1$. To do this, we loop the diagram four times, obtaining

$$
\begin{aligned}
& K\left(Z_{2}, 5\right) \\
& \quad \downarrow j \tilde{j} \\
& \Omega^{4} E \\
& \quad{ }^{\tilde{p}} \\
& K(Z, 4) \stackrel{\tilde{g}}{\longrightarrow} K\left(Z_{2}, 6\right)
\end{aligned}
$$

Since $\mathrm{Sq}^{5} l_{4}=\mathrm{Sq}^{2,1} \mathrm{Sq}^{2} l_{4}$, there exists $\tilde{v}_{0,2} \in H^{8}\left(\Omega^{4} E\right)$ such that $\tilde{j}^{*}\left(\tilde{v}_{0,2}\right)=$ $\mathrm{Sq}^{2,1} l_{5}$ and $\bar{\Delta}\left(\tilde{v}_{0,2}\right)=\tilde{p}^{*}\left(l_{4}\right) \otimes \tilde{p}^{*}\left(l_{4}\right)$. So $\bar{\Delta}\left(\mathrm{Sq}^{4} \tilde{v}_{0,2}\right)=\bar{\Delta}\left(\tilde{p}^{*}\left(l_{4}\right)^{3}\right)$. Thus

$$
\begin{aligned}
& \mathrm{Sq}^{4} \tilde{v}_{0,2}+\tilde{p}^{*}\left(l_{4}\right)^{3}+\left(\sigma^{*}\right)^{4}\left(\tilde{v}_{0,3}\right) \in P H^{12}\left(\Omega^{4} E\right) \cap \operatorname{ker}\left(\tilde{j}^{*}\right) \\
& \subset \tilde{p}^{*} P H^{12}(K(Z, 4))=0 .
\end{aligned}
$$


We have

$$
\begin{aligned}
c \tilde{p}^{*}\left(l_{4}\right)^{4} & =c \mathrm{Sq}^{8,4} \tilde{p}^{*}\left(l_{4}\right) \\
& =\left(\mathrm{Sq}^{9}+\mathrm{Sq}^{7,2}\right)\left(\sigma^{*}\right)^{4} v_{1,1}+\mathrm{Sq}^{4}\left(\sigma^{*}\right)^{4} \tilde{v}_{0,3} \\
& =\mathrm{Sq}^{7,2}\left(\sigma^{*}\right)^{4} v_{1,1}+\mathrm{Sq}^{4}\left(\sigma^{*}\right)^{4} \tilde{v}_{0,3} \\
& =\mathrm{Sq}^{7,2}\left(\sigma^{*}\right)^{4} v_{1,1}+\mathrm{Sq}^{4} \mathrm{Sq}^{4} \tilde{v}_{0,2}+\mathrm{Sq}^{4} \tilde{p}^{*}\left(l_{4}\right)^{3}
\end{aligned}
$$

Let $f: K(Z, 2) \rightarrow K(Z, 4)$ be defined by $f^{*}\left(l_{4}\right)=l_{2}^{2}$. Let $\hat{f}: K(Z, 2) \rightarrow \Omega^{4} E$ be a lifting of $f$. Then

$$
\begin{aligned}
c l_{2}^{8} & =\hat{f}^{*}\left(\mathrm{Sq}^{7,2}\left(\sigma^{*}\right)^{4} v_{1,1}+\mathrm{Sq}^{4} \mathrm{Sq}^{4} \tilde{v}_{0,2}+\mathrm{Sq}^{4} \tilde{p}^{*}\left(l_{4}\right)^{3}\right) \\
& =0+\lambda \mathrm{Sq}^{4,4} l_{2}^{4}+\mathrm{Sq}^{4} l_{2}^{6}, \quad \lambda=0 \text { or } 1 \\
& =l_{2}^{8} .
\end{aligned}
$$

So $c=1$.

\section{Relations in $P_{2} X$}

Henceforth, we will assume that $X$ is an $H$-space with $\bmod 2$ cohomology,

$$
H^{*}(X)=Z_{2}\left[x_{1}, \ldots, x_{d}\right] \otimes \Lambda\left(y_{1}, \ldots, y_{d}\right)
$$

as in the statement of Theorem A. For degree reasons, $H^{*}(X)$ is a primitively generated Hopf algebra.

According to [1], there is an exact triangle

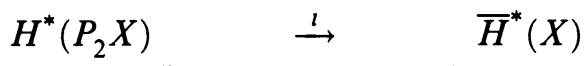

$$
\begin{aligned}
& \lambda_{\bar{H}^{*}}(X) \otimes \bar{H}^{*}(X){ }^{/ \bar{\Delta}},
\end{aligned}
$$

in which $l$ has degree $-1, \lambda$ has degree 2 , and $\bar{\Delta}$ has degree 0 .

Therefore there exist unique elements $u_{i} \in H^{5}\left(P_{2} X\right)$ and $v_{i} \in H^{6}\left(P_{2} X\right)$ such that $l\left(u_{i}\right)=x_{i}, l\left(v_{i}\right)=y_{i}$, and $\mathrm{Sq}^{1} u_{i}=v_{i}$. A simple calculation following [1] shows that

$$
\frac{Z_{2}\left[u_{1}, \ldots, u_{d}, v_{1}, \ldots, v_{d}, \mathrm{Sq}^{4} u_{1}, \ldots, \mathrm{Sq}^{4} u_{d}\right]}{(\ldots)^{3}} \subset H^{*}\left(P_{2} X\right) .
$$

Note that $\mathrm{Sq}^{2} H^{6}\left(P_{2} X\right)=0$. Using the Adem relation $\mathrm{Sq}^{2,3}=\mathrm{Sq}^{5}+\mathrm{Sq}^{4,1}$ and the fact that $\mathrm{Sq}^{3} u_{i}=0$, we obtain

$$
\mathrm{Sq}^{4} v_{i}=\mathrm{Sq}^{4,1} u_{i}=\mathrm{Sq}^{5} u_{i}=\mathrm{Sq}^{1,4} u_{i}
$$

Hence, if $\rho: H^{*}\left(P_{2} X ; Z\right) \rightarrow H^{*}\left(P_{2} X ; Z_{2}\right)$ is $\bmod 2$ reduction, we have

$$
\mathrm{Sq}^{4} H^{6}\left(P_{2} X ; Z_{2}\right) \subset \operatorname{Im}(\rho) \text {. }
$$


Consider the following commutative diagram of infinite loop maps

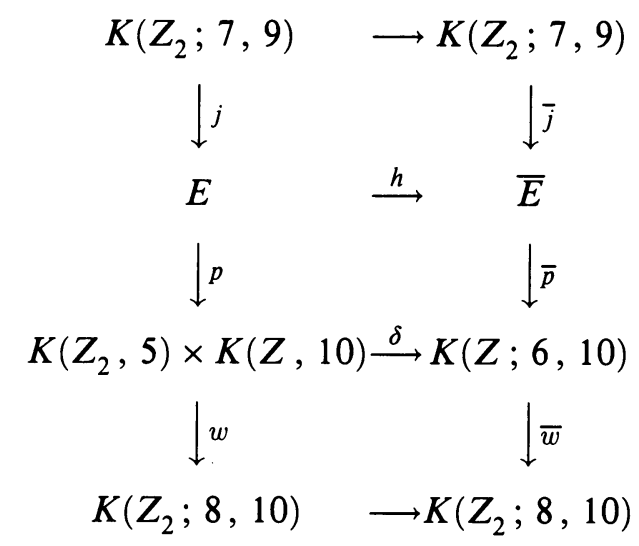

in which

$$
\begin{aligned}
& \bar{w}^{*}\left(l_{8}\right)=\mathrm{Sq}^{2} l_{6} ; \quad \bar{w}^{*}\left(l_{10}\right)=\mathrm{Sq}^{4} l_{6}-p\left(l_{10}\right) ; \\
& w^{*}\left(l_{8}\right) \mathrm{Sq}^{2} \mathrm{Sq}^{1} l_{5} ; \quad w^{*}\left(l_{10}\right)=\mathrm{Sq}^{4} \mathrm{Sq}^{1} l_{5}-p\left(l_{10}\right) ;
\end{aligned}
$$

and

$$
\delta^{*}\left(l_{10}\right)=l_{10} ; \quad \delta^{*}\left(l_{6}\right)=\operatorname{Sq}^{1} l_{5} .
$$

There exist elements $\bar{v}_{1,1} \in P H^{9}(\bar{E}), \bar{v}_{0,2} \in P H^{10}(\bar{E})$ such that $\bar{j}^{*}\left(\bar{v}_{1,1}\right)=$ $\mathrm{Sq}^{2} l_{7}$ and $\bar{j}^{*}\left(\bar{v}_{0,2}\right)=\mathrm{Sq}^{2,1} l_{7}+\mathrm{Sq}^{1} l_{9}$.

Lemma 2.1. If $v_{1,1}=h^{*}\left(\bar{v}_{1,1}\right)$ and $v_{0,2}=h^{*}\left(\bar{v}_{0,2}\right)$, then $\mathrm{Sq}^{2} v_{1,1}=\mathrm{Sq}^{1} v_{0,2}$. Proof. Note that

$$
\mathrm{Sq}^{2} \bar{v}_{1,1}+\mathrm{Sq}^{1} \bar{v}_{0,2} \in \operatorname{ker} \bar{j}^{*}=\bar{p}^{*}\left(P H^{11}(K(Z ; 6,10))\right)=0 .
$$

Therefore $\mathrm{Sq}^{2} \bar{v}_{1,1}=\mathrm{Sq}^{1} \bar{v}_{0,2}$. Applying $h^{*}$ gives the lemma.

Define $\varphi_{1,1}$ and $\varphi_{0,2}$ to be the secondary operations defined by the elements $v_{1,1}$ and $v_{0,2}$, respectively. We note that if $y \in H^{5}\left(P_{2} X\right)$, then $\varphi_{1,1}$ and $\varphi_{0,2}$ are defined on $\mathrm{Sq}^{1} y$ with zero indeterminacy.

We have the following relations:

(1) $\varphi_{1,1} \mathrm{Sq}^{1} H^{5}\left(P_{2} X\right) \subset \mathrm{Sq}^{4} H^{5}\left(P_{2} X\right)$, since $H^{9}\left(P_{2} X\right)=\mathrm{Sq}^{4}\left(P_{2} X\right)$.

(2) $\operatorname{Sq}^{2} \varphi_{1,1} \mathrm{Sq}^{1} H^{5}\left(P_{2} X\right)=\mathrm{Sq}^{1} \varphi_{0,2} \mathrm{Sq}^{1} H^{5}\left(P_{2} X\right)$, by Lemma 2.1 .

Proposition 2.2. $\varphi_{0,2} \mathrm{Sq}^{1} H^{5}\left(P_{2} X\right) \subset \mathrm{Sq}^{5} H^{5}\left(P_{2} X\right)$. 
Proof. Let $y$ be in $H^{5}\left(P_{2} X\right)$. Then

$$
\begin{aligned}
\mathrm{Sq}^{1} \varphi_{0,2} \mathrm{Sq}^{1} y & =\mathrm{Sq}^{2} \varphi_{1,1} \mathrm{Sq}^{1} y \text { by Lemma } 2.1 \\
& =\mathrm{Sq}^{2} \mathrm{Sq}^{4} y^{\prime} \text { by } 1 \\
& =\mathrm{Sq}^{5} \mathrm{Sq}^{1} y^{\prime} \\
& =\mathrm{Sq}^{1} \mathrm{Sq}^{4} \mathrm{Sq}^{1} y^{\prime} \\
& =\mathrm{Sq}^{1} \mathrm{Sq}^{1} \mathrm{Sq}^{4} y^{\prime} \\
& =0 .
\end{aligned}
$$

But $H^{10}\left(P_{2} X\right) \cap \operatorname{kerSq}^{1} \subset \operatorname{Sq}^{5} H^{5}\left(P_{2} X\right)$.

In the proofs of the next two theorems we will be using properties of the $c$-invariant and the $H$-deviation. We refer the reader to the appendix of [5] for a summary of general properties of these operations.

Theorem 2.3. $\varphi_{1,1} \mathrm{Sq}^{1} H^{5}\left(P_{2} X\right)=0$.

Proof. We now construct a Postnikov system which reflects the following relations for $u \in H^{5}\left(P_{2} X\right)$ :

(1) $\varphi_{1,1} \mathrm{Sq}^{1} u=\mathrm{Sq}^{4} u^{\prime}$,

(2) $\varphi_{0,2} \mathrm{Sq}^{1} u=\mathrm{Sq}^{5} u^{\prime \prime}$.

Consider the diagram

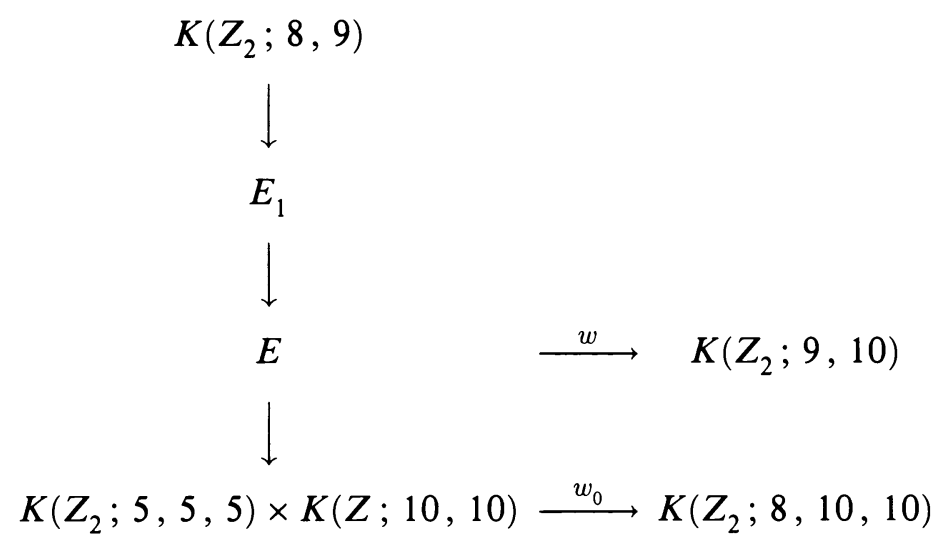

in which

$$
w_{0}^{*}\left(l_{8}\right)=\mathrm{Sq}^{2,1} l_{5}, \quad w_{0}^{*}\left(l_{10}\right)=\mathrm{Sq}^{4,1} l_{5}-l_{10}, \quad w_{0}^{*}\left(l_{10}^{\prime}\right)=\mathrm{Sq}^{4,1} l_{5}^{\prime}-l_{10}^{\prime},
$$

and

$$
w^{*}\left(l_{9}\right)=\varphi_{1,1} \mathrm{Sq}^{1} l_{5}-\mathrm{Sq}^{4} l_{5}^{\prime}, \quad w^{*}\left(l_{10}\right)=\varphi_{0,2} \mathrm{Sq}^{1} l_{5}-\mathrm{Sq}^{5} l_{5}^{\prime \prime} .
$$

We shall retain the notation $l_{5}$ and $l_{5}^{\prime}$ to denote the images of these elements in $E$ and $E_{1}$. 
In $H^{*}\left(E_{1}\right)$ we have

$$
\begin{aligned}
\mathrm{Sq}^{6} l_{5}^{\prime} & =\left(\mathrm{Sq}^{2} \mathrm{Sq}^{4}+\mathrm{Sq}^{5} \mathrm{Sq}^{1}\right) l_{5}^{\prime} \\
& =\mathrm{Sq}^{2} \mathrm{Sq}^{4} l_{5}^{\prime}+\mathrm{Sq}^{1}\left(\mathrm{Sq}^{4} \mathrm{Sq}^{1} l_{5}^{\prime}\right) \\
& =\mathrm{Sq}^{2} \varphi_{1,1} \mathrm{Sq}^{1} l_{5}^{\prime}+\mathrm{Sq}^{1} l_{10}^{\prime} \\
& =\mathrm{Sq}^{1} \varphi_{0,2} \mathrm{Sq}^{1} l_{5} \\
& =\mathrm{Sq}^{1} \mathrm{Sq}^{5} l_{5}^{\prime \prime} .
\end{aligned}
$$

This Postnikov system can be delooped. It follows that there is an element $v \in H^{10}\left(E_{1}\right)$ with $j_{1}^{*}(v)=\operatorname{Sq}^{2} l_{8}+\operatorname{Sq}^{1} l_{9}^{\prime}$ and $\bar{\Delta} v=l_{5}^{\prime} \otimes l_{5}^{\prime}$. Therefore $c\left(\sigma^{*} v\right)=$ $\sigma^{*}\left(l_{5}^{\prime}\right) \otimes \sigma^{*}\left(l_{5}^{\prime}\right)$. Since $P_{2} X$ lifts to $E_{1}$, there is an $H$-lifting $f_{1}$ of $X$ to $\Omega E_{1}$. Checking degrees, one sees that $X$ lifts to $\Omega E_{1}$ by a $c$-map. This implies that

$$
c\left(f_{1}^{*} \sigma^{*} v\right)=x^{\prime} \otimes x^{\prime} \neq 0, \quad \text { where } u^{\prime}=i\left(x^{\prime}\right) .
$$

Therefore $f_{1}^{*} \sigma^{*} v$ is a nonzero primitive in $H^{9}(X)$. But $P H^{9}(X)=0$.

\section{Proof of Theorem A}

We can now prove Theorem A.

Proof. By (2.2), if $u \in H^{5}\left(P_{2} X\right)$, then $\left(\mathrm{Sq}^{4} u\right)^{2} \neq 0$. We have

$$
\begin{aligned}
\left(\mathrm{Sq}^{4} u\right)^{2} & =\mathrm{Sq}^{9,4} u \\
& =\mathrm{Sq}^{8,4,1} u \quad \text { since } \mathrm{Sq}^{2} u=0 \\
& =\mathrm{Sq}^{4} \tilde{\varphi}_{0,3} \mathrm{Sq}^{1} u+\left(\mathrm{Sq}^{9}+\mathrm{Sq}^{7,2}\right) \varphi_{1,1} \mathrm{Sq}^{1} u \quad \text { by Theorem } 1.1 \\
& =\mathrm{Sq}^{4} \tilde{\varphi}_{0,3} \mathrm{Sq}^{1} u \quad \text { by Theorem } 2.3 .
\end{aligned}
$$

Consider the following Postnikov system:

$$
\begin{aligned}
& K\left(Z_{2} ; 8,13\right) \\
& \downarrow j_{1} \\
& E_{1} \\
& p_{1} \downarrow \\
& E \stackrel{w_{1}}{\longrightarrow} K\left(Z_{2} ; 9,14\right) \\
& \downarrow r \\
& K\left(Z_{2}, 5\right) \stackrel{w_{0}}{\longrightarrow} K\left(Z_{2} ; 7,8\right)
\end{aligned}
$$

in which

$$
\begin{aligned}
& w_{0}^{*}\left(l_{7}\right)=\mathrm{Sq}^{2} l_{5} ; \quad w_{0}^{*}\left(l_{8}\right)=\mathrm{Sq}^{2,1} l_{5} ; \\
& w_{1}^{*}\left(l_{9}\right)=\varphi_{1,1} \mathrm{Sq}^{1} l_{5} ; \quad w_{1}^{*}\left(l_{14}\right)=\tilde{\varphi}_{0,3} \mathrm{Sq}^{1} l_{5} \text {. }
\end{aligned}
$$


In $H^{*}\left(E_{1}\right)$,

$$
\begin{aligned}
\left(\mathrm{Sq}^{4} l_{5}\right)^{2} & =\mathrm{Sq}^{8,5} l_{5} \\
& =\mathrm{Sq}^{8,4,1} l_{5} \\
& =\mathrm{Sq}^{4} \tilde{\varphi}_{0,3} \mathrm{Sq}^{1} l_{5}+\left(\mathrm{Sq}^{9}+\mathrm{Sq}^{7,2}\right) \varphi_{1,1} \mathrm{Sq}^{1} l_{5} \\
& =0 .
\end{aligned}
$$

Therefore in $H^{16}\left(\Omega E_{1}\right)$ there exists an element $v$ with

$$
\begin{aligned}
\left(\Omega j_{1}\right)^{*}(v) & =\mathrm{Sq}^{4} l_{12}+\left(\mathrm{Sq}^{9}+\mathrm{Sq}^{7,2}\right) l_{7}, \\
\bar{\Delta} v & =l_{4}^{2} \otimes l_{4}^{2} .
\end{aligned}
$$

We have the following commutative diagram

$$
K\left(Z_{2} ; 7,12\right)
$$

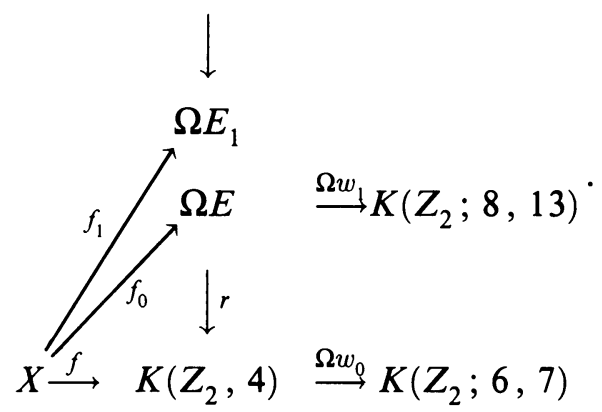

Here $f$ is an $H$-map, since the relations defined by $w_{0}$ hold in $P_{2} X$. Further, $c(f)$ factors through $K\left(Z_{2} ; 4,5\right)$, so in fact $f$ is a $c$-map. It follows that $\left(1-T^{*}\right)\left[D_{f_{1}}\right]=\Omega w_{1} \circ c(f) \simeq *$. So $\left[D_{f_{1}}\right] \in H^{12}(X \Lambda X) \cap \operatorname{ker}\left(1-T^{*}\right)$.

Any terms of the form $x_{i} \otimes x_{j}^{2}+x_{j}^{2} \otimes x_{i}$ may be eliminated by changing $f_{1}$ by $x_{i} x_{j}^{2}$. It follows that

$$
\left[D_{f_{i}}\right]=\sum_{j \neq k} b_{i j k}\left(x_{i} \otimes x_{j} x_{k}+x_{j} x_{k} \otimes x_{i}\right), \quad b_{i j k} \in Z_{2} .
$$

Therefore

$$
\bar{\Delta} f_{1}^{*}(v)=x^{2} \otimes x^{2}+\mathrm{Sq}^{4} \Sigma b_{i j k}\left(x_{i} \otimes x_{j} x_{k}+x_{j} x_{k} \otimes x_{i}\right) \neq 0 .
$$

This implies that if $\left\langle t, x^{2}\right\rangle \neq 0$, then $t^{2} \neq 0$ for $t \in H_{*}(X)$. But all squares are zero in $H_{*}(X)$, since $H_{*}(X)$ is primitively generated. We conclude that $X$ is not homotopy-commutative.

\section{REFERENCES}

1. W. Browder and E. Thomas, On the projective plane of an $H$-space, Ill. J. Math. 7 (1963), 492-502.

2. J. Harper, On the cohomology of stable two-stage Postnikov systems, Trans. Amer. Math. Soc. 152 (1970), 375-388. 
3. J. Hubbuck, On homotopy commutative $H$-spaces, Topology 8 (1969), 119-126.

4. J. Lin, A cohomological proof of the torus theorem, Math. Z. 190 (1985), 469-476.

5. J. Lin and F. Williams, On 6-connected finite $H$-spaces with two torsion, Topology 28 (1989), 7-34.

6. M. Slack, Maps between iterated loop spaces, J. Pure Appl. Algebra (to appear).

7. $\ldots$ A classification of homotopy commutative finitely generated $H$-spaces, Mem. Amer. Math. Soc. (to appear).

8. H. Toda, Composition methods in homotopy groups of spheres, Ann. of Math. Studies No. 49, Princeton Univ. Press, Princeton, NJ, 1961.

Department of Mathematics, University of California at San Diego, la Jolla, CaliFORNIA 92093 88001

Department of Mathematics, New Mexico State University, Las Cruces, New Mexico 